\title{
BOHLER'S ANGLE: CORRELATION WITH OUTCOME IN DISPLACED INTRA- ARTICULAR CALCANEAL FRACTURES TREATED WITH LOCKING COMPRESSION PLATE FIXATION WITH AND WITHOUT BONE GRAFTING
}

Deepak P. Agrawal ${ }^{1}$, Eknath D. Pawar², Vijayanand Lokhande 3 , Satish Goyal ${ }^{4}$

\section{HOW TO CITE THIS ARTICLE:}

Deepak P. Agrawal, Eknath D. Pawar, Vijayanand Lokhande, Satish Goyal. "Bohler's Angle: Correlation with Outcome in Displaced Intra-Articular Calcaneal Fractures Treated with Locking Compression Plate Fixation with and without Bone Grafting". Journal of Evolution of Medical and Dental Sciences 2014; Vol. 3, Issue 45, September 18; Page: 10946-10953, DOI: 10.14260/jemds/2014/3429

ABSTRACT: BOHLER'S ANGLE: correlation with outcome in displaced intra-articular calcaneal fractures Treated with locking compression Plate Fixation with and without bone grafting. AIMS: The aim is an accurate reduction of the fracture with reconstruction of Bohler's angle, length and axis and sub talar joint surface. To determine whether autologous bone graft supplementation is beneficial in achieving and maintaining restoration of Calcaneal height and anatomic reduction. SETTINGS AND DESIGN: Level 1 trauma center, Prospective, randomized. METHODS AND MATERIAL: Consecutive 46 patients who had fracture calcaneum were treated by open reduction and internal fixation by locking plate with and without bone graft during the period from November 2009 to April 2012. STATISTICAL ANALYSIS USED: AOFAS-Ankle-Hind foot Scale, t Test. RESULTS: Fewer complications and statistically significant better results related to treatment with locking plates with bone grafting confirmed in comparison to without bone grafting ones were noted for intra-articular calcaneal fractures. In Group A the mean time for union was 10.39 wks. The results were good and excellent in 86.95\%, $8.69 \%$ had fair result and 4.34\% had poor results. In Group B the mean time for union was 11.95 wks. The overall results were good and excellent in $73.91 \%, 13.04 \%$ had fair result and 13.04 $\%$ had poor results. CONCLUSIONS: The operative treatment of intra-articular calcaneal fractures could restore Böhler's angle better and the patient could return to full weight bearing earlier. We confirmed that autologous bone graft supplementation is beneficial in achieving and maintaining restoration of calcaneal height and anatomic reduction.

KEYWORDS: low profile locking plate, intra-articular calcaneal fracture, Bohler's angle, early weight. KEYMESSAGES: Autologous bone graft supplementation is beneficial in achieving and maintaining restoration of Bohler's angle.

INTRODUCTION: Calcaneum is the commonest tarsal bone to be fractured1. Injuries to calcaneum are caused by falling from a height and landing on foot or motor vehicle accidents. It is common in young adults engaged in building construction work and in those climbing trees and electric pole. Calcaneal fractures are one of the most disabling fractures in men, with frequent occurrence during the wage-earning period of life.

The rehabilitation process can be time-consuming, and take up to 9 months and even longer in 20 percent of patients2. Calcaneal fractures are a large economic burden to society. The functional sequelae of the foot are serious, and therapy is economically demanding. Calcaneal fractures are relatively rare injuries, with reported occurrence of $2 \%$ of all fractures. 
The intra-articular types constitute to $75 \%$ of calcaneal fractures3. $70 \%$ has involvement of the posterior subtalar joint, and approximately $80 \%$ of all fractures occur in male patients. Calcaneal fractures are rare in childhood; only $5 \%$ of all calcaneal fractures are seen in children.

TREATMENT MODALITIES: The treatment of calcaneal fracture has long been major orthopaedic problem. Because of its unique shape, difficulties arose in understanding the geometry of the fracture. Because of its location, surgical treatment was fraught with complication. ${ }^{14}$ Recently with the development of computerized tomography (CT) scanning with 3D reconstruction, the anatomy and pathology of this fracture has been understood.5,6

The imaging technique has revolutionized the treatment of calcaneum fracture. There is a considerable amount of literature on calcaneal fractures and their treatment; however the best management approach has yet to be determined. The treatment of calcaneal fractures is complex and has to be individualized depending on patient characteristics, fracture type and the condition of the soft tissues. $8,9,17$

The therapeutic modalities for displaced intra-articular calcaneal fractures can be divided into conservative and operative management.11,12 The latter comprises both the open reduction and internal fixation (ORIF) and percutaneous reduction and internal fixation (PRIF). ${ }^{15,16}$ Since the midnineties, ORIF is considered the gold standard treatment for displaced intraarticular20 fractures of the calcaneus by most experts, as it generally provides overall good to excellent results and the ability to anatomically restore the subtalar joint.

Good results are achieved if the stable anatomical reduction of the posterior facet is possible. Several open surgical techniques have been described in the past, of which the extended lateral approach has been applied most frequently. Alternative operative techniques include a medial approach, plantar approach, combined lateral and medial approach, limited posterior approach, and the sinus tarsi approach.

The results of ORIF and conservative treatment have been described and compared in several studies.11,12 These studies show improved outcome after operative treatment in subgroups and a higher rate of failed initial treatment with an increased need for a subtalar arthrodesis in conservatively treated patients. ${ }^{13,18}$

The calcaneus has been compared to an egg, with its cortical shell being cored with cancellous bone. It is this cancellous core that is usually compressed during calcaneal fracture. It has been a common practice during open reduction and internal fixation for fracture of the calcaneus to supplement the fixation with bone graft, most commonly using an auto graft from the iliac crest. This practice aims to fill in the empty core of the calcaneus to facilitate fracture healing. While morbidity at the donor site from iliac crest auto graft is well recognized. ${ }^{21,22}$

Bohler's tuber joint angle 7 is commonly assessed when evaluating calcaneal fractures. A severe heel fracture will result in a significant decrease or loss of this angle. At present, there is only a limited amount of data available on the outcome after open reduction internal fixation of calcaneal fractures. However, most available studies lack sufficient patient numbers or duration of follow-up.

The purpose of this study was to evaluate the correlation between Bohler's angle and functional outcome in displaced intra-articular calcaneal fractures Treated with low profile locking plate Fixation with and without bone grafting. 


\section{SUBJECTS AND METHODS:}

INCLUSION CRITERIA: Included in this study were all patients that fulfilled our department's general criteria for surgical therapy of calcaneal fractures: Age more than 18 years and less than 65 years, Deformation of the calcaneus, the flattening of the foot (Bohler's angle), and the intra articular fracture type.

EXCLUSION CRITERIA: Contraindications were general or local contraindications to surgery in general, a biological age of more than 65 years, extra-articular fractures, compound calcaneal fracture, peripheral vascular disease and patients who might have been unwilling or unable to follow the postoperative regime.

We operated 38 males (90.47\%) and 04 females (9.52\%) with an average age of 33.59 years (18-65 years). 46 fractures Treated with low profile locking plate Fixation with and without bone grafting. Patients treated with low profile locking plate Fixation with bone grafting were considered in Group A and patients treated with low profile locking plate Fixation without bone grafting were considered in Group B.

Whether patients will undergo bone grafting or not was decided in a randomized manner. Out of the total 46 patients; 23 patients were included in each group. The most frequent cause of accident was a fall $(\mathrm{n}=33)(78.57 \%)$ from an average height of up to $3 \mathrm{~m}$. Traffic accidents were seen in 08 cases $(19.04 \%)$ and assault in 01 case $(2.38 \%)$.

According to the classification by Essex-Lopresti19, predominantly joint depression type fractures occurred $(n=30)(65.21 \%)$, with 16 cases $(34.78 \%)$ of tongue type fractures. .05 cases $(11.90 \%)$ were associated with additional injuries, 04 patients $(9.52 \%)$ had suffered fractures of the spinal column.

Surgical treatment of the fractures took place once soft tissue conditions allowed. The average duration from admission to surgery was 8 days (5-14days).the mean operative time was $1 \mathrm{hr} 45$ minutes (1hr $25 \mathrm{~min}-2 \mathrm{hr} 55 \mathrm{~min}$ ). In all cases a fluoroscopy was performed after reduction and temporary fixation of the fracture. Postoperatively radiographs were taken; we did not regularly perform CT scan postoperatively.

All patients had pre- and postoperative X-Rays, so the change of the Bohler's angle and the length of the hind foot could be measured exactly. Clinical follow-up examination was performed at 4 weeks, 6 weeks, 10 weeks, 3 months, 6 months and 1 year.

SURGICAL TECHNIQUE: Patients were operated under spinal/ general anesthesia. The patient is positioned in a lateral decubitus position. Pneumatic tourniquet/ Esmarch rubber tourniquet was used in all patients. Patients were positioned laterally on an operating table with a radiolucent carbon part. An extended lateral approach was performed. The approach was developed as a fullthickness flap.

The lateral cortical fragment (bulge fragment) was then hinged away. Subsequently, a good view into the sub talar joint was obtained. The soft tissue flap was held back by K-wires, which had been inserted into the talus and bent. Use of the joy-stick technique with a Schanz-screw placed through the tuber calcanei achieves reduction and in particular the length and axis is regained. 
The fractured lateral wall of the calcaneus is gently opened, leaving the fracture fragments within their periosteal envelope. The fragments are elevated, the articular surface is reduced. The individually reduced bone fragments were fixed temporarily with K-wires seen under C-Arm IITV.

In group A patients bone graft was taken from ipsilateral iliac bone without cutting iliac crest to decrease donor site morbidity. The bone graft consisted of both inner \& outer cortex with cancellous bone sandwiched between them. It was placed in the defect below articular surface. Bohlers angle was reconstructed and seen under C-arm. After satisfactory reduction, plate was applied and fixation of the plate was done with locked screws. Particular care is taken to fix the central fragment (posterior sub talar fragment) in a good and stable position.

The implant used was a multi-directional low profile locking plate.

STATISTICS: Statistical significance was taken as 0.05 . The t test for equality was used to test for significance of difference.

\section{Group A}

Paired Samples Statistics

Mean N Std. Deviation Std. Error Mean

Pre-op 9.52213 .763 .821

Post-op 28.90215 .8731 .282

Paired Samples Correlations

N Correlation Sig.

Pre-op \& post-op 21.500 .021

$\mathrm{P}$ value is $<0.05$ i.e. 0.021 , means there is significant difference in Bohler's angle after operation. At 20 degrees of freedom, 5\% significant limit of $t$ is 2.09.The observed $t$ value is 7.237 times the SE hence there is no doubt that surgery improves Bohler's angle.

\section{Group B}

Paired Samples Statistics

Mean N Std. Deviation Std. Error Mean

Post-op 21.10216 .1561 .343

Pre-op 10.95212 .906 .634

Paired Samples Correlations

N Correlation Sig.

Post-op \& pre-op 21.839 .000

$\mathrm{P}$ value is $<0.05$ i.e. $<0.0001$, means there is significant difference in Bohler's angle after operation. At 20 degrees of freedom, $5 \%$ significant limit of $t$ is 2.09 . The observed $t$ value is 11.50 times the SE hence there is no doubt that surgery improves Bohler's angle.

\section{Group A \& Group B}

Paired Samples Statistics

Mean N Std. Deviation Std. Error Mean

Post-op A 29.38215 .6871 .241

Post-op B 21.10216 .1561 .343 
Paired Samples Correlations

N Correlation Sig.

Post-op A \& post-op B $21-.165 .474$

At 20 degrees of freedom, 5\% significant limit of $t$ is 2.09.The observed $t$ value is 4.198 times the SE hence Group A is better than Group B.

RESULTS: Group A: In patients treated with plating with bone grafting partial weight bearing was started within 6-8 weeks and full weight bearing within 3- 6 months.13 patients $(61.90 \%)$ walked full weight bearing in 3 months duration, while 08 patients $(38.09 \%)$ took 4-6 months due to delayed wound healing or associated injuries.

The time for union was 8 - 14 wks. In 22 cases (95.65\%) normal Bohler angle (i. e. 200-400) was achieved. There were 3 cases (13.04\%) of post-operative delayed wound healing. The gaping was at the angle of the incision for $2-3 \mathrm{~cm}$.It was deep till subcutaneous tissue but plate was not exposed. All healed with daily dressing and delayed mobilization within 3 weeks and had no further treatment. 01 patients $(4.34 \%)$ had superficial wound infection and were been treated with oral antibiotics. None of the patients developed severe infection.

None of the patient developed sural nerve complication in the form of loss of sensation on lateral aspect of foot or had painful hardware. The overall results according to the AOFAS Ankle Hind foot Scale were good and excellent in 20/23 (86.95\%), 4 (17.39\%) of the patients had excellent results, $16(69.56 \%)$ had good results, 2 (8.69\%) had fair result and $1(4.34 \%)$ had poor results.

Group B: In group B patients treated with plating without bone grafting, partial weight bearing was started within 6-8 weeks and full weight bearing within 3- 6 months.10 patients (47.61\%) walked full weight bearing in 3 months duration, while 11 cases (52.38\%) took 4-6 months due to delayed wound healing or associated injuries.

The average time for union was 10- 16 wks. In 17 cases (73.91\%) normal Bohler angle (i. e. 200-400) was achieved. There were 3 cases (13.04\%) of operative delayed wound healing. It was deep till subcutaneous tissue but plate was not exposed. All healed with daily dressing and delayed mobilization within 3 weeks and had no further treatment. 01 patient (4.34\%) had superficial wound infection and treated with oral antibiotics.

None of the patients developed severe infection. 01(4.34\%) of the patient developed sural nerve complication in the form of loss of sensation on lateral aspect of foot and 01(4.34\%) had painful hardware, so implant removal was done after bony union. The overall results according to the AOFAS Ankle Hind foot Scale were good and excellent in 17/23 (73.91\%).3/23 (13.04\%) had fair result and 3/23(13.04 \%) had poor results.

\section{Table: 1}

\section{Group-A Group-B}

Mean Partial weight bearing (weeks) 6.476 .61

Mean Full weight bearing (months) 3.093 .95

Mean Pre-operative Bohler angle 10.08010 .950

Mean Post-operative Bohler angle 29.080 21.430

Mean Bohler angle at the time of Union 28.43018 .260

Mean Union (weeks) 10.3911 .95 


\section{ORIGINAL ARTICLE}

Delayed wound healing 33

Superficial wound infection 11

Painful hardware 01

Sural nerve complication 01

There is statistically significant difference between the bone graft and non-bone graft patients regarding the change in Böhler's angle obtained with surgery .we confirmed that autologous bone graft supplementation is beneficial in achieving and maintaining restoration of calcaneal height and anatomic reduction.

DISCUSSION: Although surgical treatment of displaced calcaneal fractures is an established surgical standard. It is not clear, if accurate anatomic reduction is of major importance. Literature showed that fracture volume treated by an institution is an independent determinant for the postoperative infection rate and for the development of subtalar arthritis. The restoration of the tension of the plantar fascia by reconstruction of Bohlers angle and hind foot length is important for the biomechanics of the foot.

CT diagnostic provided improved understanding of calcaneal fractures and led to a clinically relevant classification of these injuries. Thus a better surgical planning became possible. Anatomical and biomechanical studies refer to the important role of the posterior joint facet, which has to be stabilized to reconstruct the subtler joint. Incidence and severity of posttraumatic sub talar arthrosis depends on the fracture type, calcaneal shape, and position after the osteosynthesis, chondral injury of the subtalar joint, and articular facet congruency.

Special plates have been invented to suit the needs for the treatment of calcaneal fractures (e.g. AO calcaneal plate, Galveston plate, low contact plate). The selected implant should be able to neutralize the forces resulting from the Achilles tendon and maintain reduction of the fragments until bony consolidation. Because of the variety of holes each fracture can be treated individually. Especially the main fragment (posterior fragment) can be fixed safely.

The operative treatment of intra articular calcaneum fractures with/without bone graft is a still a topic of debate. Many studies had reported higher infection rate in the treatment of intra articular calcaneum fractures with bone graft and this was one of the reason for the authors to propagate no use of bone graft.

In our study we observed lesser infection rate in bone graft group than without bone graft group there is also concern of donor site morbidity associated with iliac crest bone graft but we were able to decrease the donor site morbidity by preserving the iliac crest. Restoration of Bohler angle was associated with better outcome. In our study post-operative Bohler angle was significantly higher in bone graft group.

Restoration of Bohler angle was statistically significantly better in the bone graft group than non-bone graft group. We have confirmed that in treatment of intra articular calcaneum fractures bone graft augmentation could restore the Bohler angle better and prevent late collapse. The patients with bone grafts could return to full weight bearing earlier. 


\section{REFERENCES:}

1. Zwipp H, Rammelt S, Barthel S. Fracture of the calcaneus. Unfallchirurg 2005; 108: 737-48.

2. Tufescu TV, Buckley R. Age, gender, work capability, and worker's compensation in patients with displaced intra articular calcaneal fractures. J Orthop Trauma 2001; 15: 275-9.

3. Lindsay WRN, Dewar FP. Fractures of the os calcis. Am J Surg 1958; 95: 555, 576.

4. Sanders R (2000) Displaced intra-articular fractures of the calcaneus. J Bone Joint Surg Am 82 (2): 225-250.

5. Crosby LA, Fitzgibbons T. Computerized tomography scanning of acute intra-articular fractures of the calcaneus. J Bone Joint Surg 1990; 72: 852-859.

6. Aditya Daftary, Andrew H. Haims, Michael R. Baumgaertner,: Fractures of the Calcaneus: A Review with Emphasis on CT. Radio Graphics, 25:1215-1226,2005.

7. Bohler L.; Diagnosis, pathology and treatment of fractures of the os calcis; J Bone Joint Surg 1931; 13: 75-89.

8. Palmer I. The mechanism and treatment of fractures of the calcaneus. J Bone Joint Surg Am 1948; 30: 2-8.

9. Conn HR; The treatment of fractures of the os calcis. J Bone Joint Surg 1935; 17: 392-405.

10. Zwipp H, Tscherne $\mathrm{H}$, Thermann $\mathrm{H}$, Weber T. Osteosynthesis of displaced intra articular fractures of the calcaneus. Clin Orthop Relat Res 1993; 290: 76-86.

11. Parmar HV, Triffitt PD, Gregg PJ. Intra-articular fractures of the calcaneum treated operatively or conservatively: a prospective study. J Bone Joint Surg Br 1993; 75: 932-937.

12. Thordarson DB, Krieger LE. Operative vs. non-operative treatment of intra-articular fractures of the calcaneus: a prospective randomized trial. Foot Ankle Int 1996; 17: 2-9.

13. Crosby LA, Fitzgibbons T. Intra articular calcaneal fractures. Results of closed treatment. Clin Orthop Relat Res. 1993; 290: 47-54.

14. Bajammal S, Tornetta P 3rd, Sanders D, Bhandari M. Displaced intra-articular calcaneal fractures. J Orthop Trauma 2005; 19: 360-4.

15. Rammelt S, Amlang M, Barthel S, et al. Minimally-invasive treatment of calcaneal fractures. Injury 2004; 35 (Suppl. 2): SB55-SB63. Foot Ankl Int 1996; 17: 2-9.

16. Schepers T, Schipper IB, Vogels LM, Ginai AZ, Mulder PG, Heetveld MJ, Patka P (2007) percutaneous treatment of displaced intra-articular calcaneal fractures. J Orthop Sci 12(1): 2227, Epub 2007 January 31.

17. Tim Alexander Walde, B. Sauer, J. Degreif, and H.-J. Walde Closed reduction and percutaneus Kirschner wire fixation for the treatment of dislocated calcaneal fractures: surgical technique, complications, clinical and radiological results after 2-10 years Arch Orthop Trauma Surg. 2008 June; 128 (6): 585-591.

18. Brunner UH, Betz A, Halama R (1991) the surgical treatment of calcaneus fractures. Orthopade 20 (1): 55-66.

19. Essex Lopresti $\mathrm{P}$. The mechanism, reduction technique, and results in fractures of the os calcis. Br J Surg 1952; 39: 395-419.

20. Zwipp H, Rammelt S, Barthel S. Calcaneal fractures-open reduction and internal fixation (ORIF). Injury 2004; 35: SB46-54.

21. Longino D, Buckley RE Bone graft in the operative treatment of displaced intraarticular calcaneal fractures: is it helpful? J Orthop Trauma.2001; 15: 280-6. 


\section{ORIGINAL ARTICLE}

22. Yang, et al, Intra-articular calcaneal fractures with or without bone grafts; A systematic review of literature. Indian j. orthp 2012; 46: 130-7.

\section{AUTHORS:}

1. Deepak P. Agrawal

2. Eknath D. Pawar

3. Vijayanand Lokhande

4. Satish Goyal

\section{PARTICULARS OF CONTRIBUTORS:}

1. Assistant Professor, Department of Orthopaedics, Government Medical College, Aurangabad.

2. Associate Professor, Department of Orthopaedics, Government Medical College, Aurangabad.

3. Resident, Department of Orthopaedics, Government Medical College, Aurangabad.

4. Assistant Professor, Department of Orthopaedics, Government Medical College, Aurangabad.

\section{NAME ADDRESS EMAIL ID OF THE CORRESPONDING AUTHOR:}

Dr. Deepak P. Agrawal, Department of Orthopedics, (K.E.M Mumbai),

Shri Gurukripa, Plot No. 133, Cidco N-3, Near High Court, Aurangabad-431003.

Email:dr_dpa@yahoo.com

Date of Submission: 12/09/2014.

Date of Peer Review: 13/09/2014.

Date of Acceptance: 15/09/2014.

Date of Publishing: 16/09/2014. 